\title{
Sur une question de H. Brezis, M. Marcus et A.C. Ponce
}

\section{On a question of H. Brezis, M. Marcus and A.C. Ponce}

\author{
Alano Ancona \\ Université Paris Sud, Centre d'Orsay, Mathématiques, Bât. 425, 91405 Orsay Cedex, France
}

Reçu le 12 octobre 2004 ; accepté le 3 février 2005

Disponible sur Internet le 15 avril 2005

\section{Résumé}

À la suite d'une question soulevée dans un travail de Brezis-Marcus-Ponce [5] on montre que si $\mu$ est une mesure de Radon diffuse et singulière sur l'ouvert $U$ de $\mathbb{R}^{d}$, il n'existe pas d'application linéaire continue $f \mapsto\left(u_{f}, g_{f}\right)$ de $L^{1}(\mu)$ dans $H^{1}(U) \times L^{1}(U)$ telle que $f \cdot \mu=\Delta u_{f}+g_{f}$ au sens de $\mathcal{D}^{\prime}(U)$. On indique ensuite une généralisation.

(C) 2006 L'Association Publications de l'Institut Henri Poincaré. Published by Elsevier B.V. All rights reserved

\section{Abstract}

Investigating a question raised in a recent paper of Brezis-Marcus-Ponce [5] we show that if $\mu$ is a singular continuous Radon measure in the open subset $U$ of $\mathbb{R}^{d}$, there is no continuous linear map $f \mapsto\left(u_{f}, g_{f}\right)$ from $L^{1}(\mu)$ to $H^{1}(U) \times L^{1}(U)$ such that $f \cdot \mu=\Delta u_{f}+g_{f}$ in the sense of distributions. A generalization is also described.

(ㅇ) 2006 L'Association Publications de l'Institut Henri Poincaré. Published by Elsevier B.V. All rights reserved

MSC : 31C15; 31C25; 35J05; 46E35

Mots-clés : Capacité; Ensemble polaire ; Espaces de Sobolev; Mesures d'énergie finie

\section{Introduction}

On considère dans cette note une des questions posées dans un travail récent (ref. [5]) de H. Brezis, M. Marcus et A.C. Ponce sur les équations non linéaires du type $-\Delta u+g(u)=\mu$ où $\mu$ est une mesure sur un domaine de $\mathbb{R}^{d}$. Cette question concerne une décomposition connue de certaines mesures et se rattache à l'étude des espaces de Sobolev du type $H^{1}(U), U$ ouvert de $\mathbb{R}^{d}$.

Adresse e-mail : alano.ancona@math.u-psud.fr (A. Ancona). 
Pour $U$ ouvert borné de $\mathbb{R}^{d}$ ( $d$ entier $\geqslant 2$ ), considérons l'espace $M(U)$ des mesures de Radon sur $U$ de masse totale finie et ne chargeant pas les ensembles polaires de la théorie classique du Potentiel (ref. [6,11]). Rappelons qu'une mesure de Radon sur $U$ qui est absolument continue par rapport à la mesure de Lebesgue ne charge pas les ensembles polaires de $U$ - on a donc $L^{1}(U) \subset M(U)$-, et que toute mesure de Radon de masse totale finie sur $U$ et de la forme $v=\Delta u$ avec $u \in H_{0}^{1}(U)$ (c'est à dire telle que $v \in H^{-1}(U)$ ) est aussi élément de $M(U)$ (cf. [10]). Inversement, Boccardo, Gallouët et Orsina ont montré que toute mesure $v$ dans $M(U)$ admet une décomposition $v=f+\Delta u$ avec $f \in L^{1}(U)$ et $u \in H_{0}^{1}(U)$ [2]. Le Théorème 3 de [5] établit, pour tout $\varepsilon>0$ donné, une telle décomposition avec $u$ continue sur $\bar{U}$, nulle sur $\partial U$, et $\|f\|_{L^{1}(U)} \leqslant\|v\|_{1},\|u\|_{H^{1}}+\|u\|_{\infty} \leqslant \varepsilon\|v\|_{1}$. Ici $\|\cdot\| \|_{1}$ désigne la norme de variation totale, soit $\|\sigma\|_{1}=|\sigma|(U)$ si $\sigma$ est une mesure de Radon sur $U$.

La question (Open Problem 3 de [5]) de Brezis, Marcus et Ponce est alors de savoir s'il existe un opérateur linéaire $v \mapsto\left(f_{v}, u_{v}\right)$ de $M(U)$ dans $L^{1}(U) \times H_{0}^{1}(U)$ tel que pour toute $v \in M(U)$ on ait $v=f_{v}+\Delta u_{v}$, cette décomposition de $v$ étant du type ci-dessus. On va voir qu'il n'en est rien - même si on n'impose pas à la décomposition les raffinements du Theorem 3 de [5] -, en établissant l'énoncé qui suit. Dans toute la suite $\lambda_{d}$ désigne la mesure de Lebesgue sur $\mathbb{R}^{d}$ et $H^{1}(U)$ l'espace de Sobolev des fonctions (réelles) $f \in L^{2}(U)$ dont le gradient $\nabla f$ (au sens des distributions) appartient à $L^{2}\left(U ; \mathbb{R}^{d}\right)$, muni du produit scalaire usuel $\langle f, g\rangle_{H^{1}(U)}=\int_{U} f g \mathrm{~d} \lambda_{d}+\int_{U} \nabla f \cdot \nabla g \mathrm{~d} \lambda_{d}$.

Théorème 1. Soit $\mu$ une mesure de Radon positive sur l'ouvert $U$ de $\mathbb{R}^{d}$ et soit

$$
K: L^{1}(\mu) \rightarrow H^{1}(U)
$$

un opérateur linéaire continu. Si pour chaque $f \in L^{1}(\mu)$ la distribution $v_{f}=\Delta(K(f))$ est une mesure de Radon sur $U$ telle que $\nu_{f}-f \cdot \mu$ soit absolument continue ( par rapport à $\lambda_{d}$ ), alors $\mu$ est nécessairement absolument continue.

Si $U$ est non vide il existe des mesures $\mu \in M(U)$ positives et singulières par rapport à $\lambda_{d}$, par exemple la mesure de $(d-1)$-volume d'une sphère (ou d'une portion d'hyperplan) contenue dans $U$. En considérant une telle mesure $\mu$, et en identifiant l'espace de Banach $L^{1}(\mu)$ à un sous-espace de $M(U)$, on voit que le théorème 1 résout négativement le problème de [5] considéré ici.

Remarque 1.1. On notera dans la partie 4 un énoncé plus général (Théorème 1bis et Corollaire 6).

Remarque 1.2. Signalons deux autres exemples connus d'opérateurs naturels, bornés et surjectifs, mais sans inverse à droite. J. Peetre [13] a montré que c'est le cas pour l'opérateur de trace $W^{1,1}\left(\mathbb{R}^{d+1}\right) \ni f \stackrel{T}{\rightarrow} f(\cdot, 0) \in$ $L^{1}\left(\mathbb{R}^{d}\right)$ de Gagliardo [9]. Si $L_{\#}^{p}:=\left\{f \in L_{\text {loc }}^{p}\left(\mathbb{R}^{d}\right) ; f 2 \pi \mathbb{Z}^{d}\right.$-périodique et $\left.\int_{[0,2 \pi]^{d}} f=0\right\}$ et si $\mathcal{E}:=\{Y \in$ $L^{\infty}\left(\mathbb{R}^{d} ; \mathbb{R}^{d}\right) ; Y 2 \pi \mathbb{Z}^{d}$-périodique et $\left.\operatorname{div}(Y) \in L_{\#}^{d}\right\}$, Bourgain et Brezis montrent dans [4] que $\mathcal{E} \ni Y \stackrel{\delta}{\mapsto} \operatorname{div}(Y) \in L_{\#}^{d}$ est surjectif mais également sans inverse à droite borné.

\section{Opérateurs linéaires $K: L^{1}(\mu) \rightarrow H^{1}(U)$ et noyaux}

Soient $U$ un ouvert de $\mathbb{R}^{d}$ et $\mu$ une mesure positive $\sigma$-finie sur un espace mesurable $(Y, \mathcal{F})$. On notera $\mathcal{B}$ or $(V)$ la tribu borélienne d'une partie $V$ de $\mathbb{R}^{d}$ et, comme plus haut, $\lambda_{d}$ la mesure de Lebesgue sur $\mathbb{R}^{d}$. La preuve du Théorème $1 \mathrm{~s}$ 'appuiera sur une description (Lemmes 2 et 3 ) des opérateurs linéaires continus $K: L^{1}(\mu) \rightarrow H^{1}(U)$, description essentiellement contenue dans un résultat classique et bien plus général de Dunford-Pettis [7] 1940 (cf. [3] p. 46, [8] p. 503). Pour des raisons de commodité de lecture, on a développé une preuve du Lemme 2 et dans une certaine mesure celle du Lemme 3. 
Lemme 2. Soit $k: U \times Y \rightarrow \mathbb{R}$ une fonction $\operatorname{Bor}(U) \otimes \mathcal{F}$-mesurable telle que, pour chaque $y \in Y, k_{y}:=k(\cdot, y) \in$ $H^{1}(U)$ et $\left\|k_{y}\right\|_{H^{1}(U)} \leqslant C$ où $C \in \mathbb{R}_{+}$est indépendant de $y$. Alors pour toute $f \in L^{1}(\mu)$, la formule

$$
K_{f}(x)=\int_{Y} k(x, y) f(y) \mathrm{d} \mu(y)
$$

définit un élément $K_{f} \in H^{1}(U)$, l'intégrale définissant $K_{f}(x)$ étant convergente au sens de Lebesgue pour $\lambda_{d}$ presque tout $x \in U$. De plus $\left\|K_{f}\right\|_{H^{1}(U)} \leqslant C\|f\|_{L^{1}(\mu)}$.

Preuve. Observons d'abord que l'application $y \mapsto k_{y}$ de $(Y, \mathcal{F})$ dans $H^{1}(U)$ est scalairement mesurable, i.e. que $y \mapsto\left\langle k_{y}, \varphi\right\rangle_{H^{1}(U)}=\int_{U} k_{y}(x) \varphi(x) \mathrm{d} x+\sum_{1 \leqslant j \leqslant d} \int_{U} \partial_{j} k_{y}(x) \partial_{j} \varphi(x) \mathrm{d} x$ est $\mathcal{F}$-mesurable pour toute $\varphi \in H^{1}(U)$. En approchant, dans $L^{2}(U), \varphi$ et les $\partial_{j} \varphi$ par des fonctions de classe $C_{0}^{\infty}(U)$, on est ramené à montrer que $y \mapsto$ $\int k(x, y) \psi(x) \mathrm{d} x$ est $\mathcal{F}$-mesurable sur $Y$ pour $\psi \in C_{0}^{\infty}(U)$. Ce qui découle du théorème de Fubini puisque la fonction $(x, y) \mapsto k(x, y) \psi(x)$ est $\operatorname{Bor}(U) \otimes \mathcal{F}$-mesurable (et $\lambda_{d}$ intégrable en $x$ ).

Comme $H^{1}(U)$ est séparable, l'application $y \mapsto k_{y}$ est donc fortement mesurable de $(Y, \mathcal{F})$ dans $H^{1}(U)$. Et comme $\left\|k_{y}\right\|_{H^{1}(U)} \leqslant C$, on voit que pour toute $f \in \mathcal{L}^{1}(\mu)$, l'intégrale vectorielle (ref. [8] III.2)

$$
\widetilde{K}_{f}:=\int_{Y} f(y) k_{y} \mathrm{~d} \mu(y)
$$

existe au sens de Bochner (c.à.d. au sens de [8] III.2) dans $H^{1}(U)$ - l'intégrante étant vue comme fonction mesurable à valeurs dans $H^{1}(U)$-, et définit un élément $\widetilde{K}_{f}$ de $H^{1}(U)$ tel que $\left\|\widetilde{K}_{f}\right\|_{H^{1}(U)} \leqslant C\|f\|_{L^{1}(\mu)}$.

Pour $\varphi \in \mathcal{L}_{\mathbb{R}}^{\infty}(U)$ à support compact, on a d'après la continuité de $u \mapsto \int_{U} u(x) \varphi(x) \mathrm{d} x$ sur $H^{1}(U)$, les propriétés de linéarité de l'intégrale vectorielle et le théorème de Fubini,

$$
\int_{U} \widetilde{K}_{f}(x) \varphi(x) \mathrm{d} x=\int_{Y} f(y)\left[\int_{U} k_{y}(x) \varphi(x) \mathrm{d} x\right] \mathrm{d} y=\int_{U} \varphi(x) K_{f}(x) \mathrm{d} x,
$$

où $\varphi K_{f}$ est définie presque partout et intégrable dans $U$. L'arbitraire sur $\varphi$ donne $K_{f}=\widetilde{K}_{f}$ p.p. dans $U$ et le lemme est établi.

Remarques 2.1. (a) On peut vérifier plus directement le lemme sans passer par la notion d'intégrale de Bochner. Mais celle-ci est utile pour la suite. (b) (Unicité de $k$ ) Deux noyaux $k^{(1)}, k^{(2)}: U \times Y \rightarrow \mathbb{R}$ comme dans le lemme définissent la même application linéaire de $L^{1}(\mu)$ dans $H^{1}(U)$ si, et seulement si, on a $k^{(1)}=k^{(2)} \lambda_{d} \otimes \mu$-p.p.

Passons à la réciproque du lemme, donnée par le théorème de Dunford-Pettis [7,3] ou [8].

Lemme 3. Soit $K: L^{1}(\mu) \rightarrow H^{1}(U)$ une application linéaire continue. Il existe une fonction $k: U \times Y \rightarrow \mathbb{R}$ qui est $\operatorname{Bor}(U) \otimes \mathcal{F}$-mesurable et telle que :

(i) pour chaque $y \in Y, k_{y}=k(\cdot, y)$ est un élément de $H^{1}(U)$ et on a $\left\|k_{y}\right\|_{H^{1}(U)} \leqslant\|K\|_{L\left(L^{1}(\mu), H^{1}(U)\right)}$,

(ii) pour chaque $f \in L^{1}(\mu)$, on a $K f(x)=\int k(x, y) f(y) \mathrm{d} \mu(y)$ pour $\lambda_{d}$ presque tout $x \in U$.

Preuve. Supposons d'abord $U$ borné. Par le théorème de Dunford-Pettis (cf. [8] p. 503 ou [3] p. 46) il existe une application $\theta: y \mapsto \theta_{y}$ de $Y$ dans $H^{1}(U)$ scalairement mesurable (et donc mesurable) telle que $\left\|\theta_{y}\right\|_{H^{1}(U)} \leqslant$ $\|K\|$ pour $y \in Y$ et $K(f)=\int_{Y} f(y) \theta_{y} \mathrm{~d} \mu(y)$ pour $f \in L^{1}(\mu)$ (intégrale de Bochner dans $H^{1}(U)$ ). Alors $\theta \in$ 
$L^{1}\left((\mu, \mathcal{F}) ; L^{1}(U)\right)^{1}$ et $L^{1}\left((\mu, \mathcal{F}) ; L^{1}(U)\right)$ s'identifie à $L_{\mathbb{R}}^{1}\left(\mathcal{B o r}(U) \otimes \mathcal{F} ; \lambda_{d \mid U} \otimes \mu\right)$, l'élément associé à $f \in$ $L_{\mathbb{R}}^{1}\left(\mathcal{B o r}(U) \otimes \mathcal{F} ; \lambda_{d \mid U} \otimes \mu\right)$ étant égal $\mu$ p.p. à $g: y \mapsto \tilde{f}(\cdot, y)$ si $\tilde{f}$ est un représentant de $f$ dans $\mathcal{L}_{\mathbb{R}}^{1}(\mathcal{B}$ or $(U) \otimes$ $\left.\mathcal{F} ; \lambda_{d \mid U} \otimes \mu\right)\left([8]\right.$, p. 196). En modifiant sur un ensemble $\mu$ négligeable convenable un élément $k \in \mathcal{L}_{\mathbb{R}}^{1}(\mathcal{B}$ or $(U) \otimes$ $\left.\mathcal{F} ; \lambda_{d \mid U} \otimes \mu\right)$ associé à $\theta$, on a, pour tout $y \in Y, \theta_{y}=k(\cdot, y)$ p.p. dans $U$. Et le noyau $k$ a bien les propriétés voulues.

Si $U$ n'est pas borné, considérons les $U_{n}=U \cap\{x \in U ;|x|<n\}, n \geqslant 0$, les $K_{n}: L^{1}(\mathcal{F}, \mu) \rightarrow H^{1}\left(U_{n}\right)$ déduits de $K$ par restriction (i.e. $K_{n}(f)=K(f)_{\mid U_{n}}$ ) et des noyaux $k_{n}: U_{n} \times Y \rightarrow \mathbb{R}$ correspondants. En choisissant $k$ égal à $k_{n} \operatorname{sur}\left(U_{n} \backslash U_{n-1}\right) \times Y$ on obtient évidemment un noyau vérifiant (i) et (ii).

Pour la suite on utilisera aussi l'observation suivante.

Lemme 4. Reprenons les hypothèses et notations du Lemme 2 et supposons de plus que pour toute $f \in L^{1}(\mu)$ le Laplacien (au sens des distributions) $\Delta K(f)$ de $K(f)$ soit une mesure de Radon dans $U$. Alors pour $\mu$ presque tout $y \in Y, \Delta k_{y}=\Delta k(\cdot, y)$ est une mesure de Radon dans $U$ et pour tout compact $L \subset U$, il existe une constante $C_{L}>0$ telle que $\left|\Delta k_{y}\right|(L) \leqslant C_{L}$ pour $\mu$-p.t. $y \in Y$. De plus si $f \in L^{1}(\mu)$ et si A est un borélien relativement compact de $U$, on a

$$
\int_{A} \mathrm{~d}[\Delta K(f)]=\int_{Y}\left[\Delta k_{y}\right](A) f(y) \mathrm{d} \mu(y) .
$$

Remarque. On verra aussi que $y \mapsto\left[\Delta k_{y}\right](A)$ est mesurable et que la dernière intégrale a bien un sens.

Preuve. (a) Soit $U^{\prime}$ un ouvert relativement compact de $U$. Pour $\varphi \in C_{0}^{\infty}(U), \lambda_{\varphi}: f \mapsto \int \varphi \mathrm{d} \Delta(K(f))=$ $\int \Delta \varphi(x) K(f)(x) \mathrm{d} x$ est une forme linéaire continue sur $L^{1}(\mu)$ et $\left\{\lambda_{\varphi}(f) ; \varphi \in C_{0}^{\infty}(U),|\varphi| \leqslant 1_{U^{\prime}}\right\}$ est borné pour chaque $f \in L^{1}(\mu)$. Le théorème de Banach-Steinhaus dit alors que

$$
C\left(U^{\prime}\right):=\sup \left\{\left|\lambda_{\varphi}(f)\right| ;\|f\|_{L^{1}(\mu)} \leqslant 1, \varphi \in C_{0}^{\infty}(U),|\varphi| \leqslant 1_{U^{\prime}}\right\}<\infty .
$$

On va dans la suite vérifier l'énoncé avec les constantes $C_{L}=\inf \left\{C\left(U^{\prime}\right) ; U^{\prime} \supset L\right\}$. Notons que $|\Delta K(f)|\left(U^{\prime}\right) \leqslant$ $C\left(U^{\prime}\right)\|f\|_{L^{1}(\mu)}$ pour toute $f \in L^{1}(\mu)$.

(b) Pour $\varphi \in C_{0}^{\infty}(U)$, on a par la définition du Laplacien au sens de $\mathcal{D}^{\prime}(U)$, et par l'expression de $K$ à l'aide du noyau $k$,

$$
\int_{U} \varphi \mathrm{d} \Delta(K(f))=\iint_{Y} \Delta \varphi(x) k_{y}(x) f(y) \mathrm{d} \mu(y) \mathrm{d} \lambda_{d}(x)=\int_{Y}\left\langle\Delta k_{y}, \varphi\right\rangle f(y) \mathrm{d} \mu(y),
$$

où par composition $y \mapsto\left\langle\Delta k_{y}, \varphi\right\rangle$ est mesurable bornée sur $Y$. Donc pour $V$ ouvert relativement compact de $U$ et $\varphi \in C_{0}^{\infty}(V),\left|\int_{Y}\left\langle\Delta k_{y}, \varphi\right\rangle f(y) \mathrm{d} \mu(y)\right| \leqslant C_{V}\|\varphi\|_{\infty}\|f\|_{L^{1}(\mu)}$. Par conséquent $\left|\left\langle\Delta k_{y}, \varphi\right\rangle\right| \leqslant C_{V}\|\varphi\|_{\infty}$ pour $\mu$ p.t. $y \in Y$. Comme $C_{0}^{\infty}(V)$ est séparable, il existe $N_{V} \in \mathcal{F} \mu$ négligeable et tel que pour tout $y \notin N_{V}$ et toute $\varphi \in C_{0}^{\infty}(V)$ on a $\left|\left\langle\Delta k_{y}, \varphi\right\rangle\right| \leqslant C_{V}\|\varphi\|_{\infty}$. Mais alors $\left(\Delta k_{y}\right)_{\mid V}$ est une mesure de Radon sur $V$ de masse totale majorée par $C_{V}$ et les premières assertions du lemme sont établies.

(c) Si $V$ est un ouvert relativement compact de $U$, une approximation de $1_{V}$ par une suite croissante d'éléments positifs de $C_{0}^{\infty}(V)$ permet d'étendre (grâce au (a)) la mesurabilité de $y \mapsto \Delta\left[k_{y}(\varphi)\right]$ et l'égalité des membres extrêmes de (2) au cas $\varphi=1_{V}$. Les parties ouvertes de tout ouvert relativement compact $U^{\prime}$ de $U$ formant un $\pi$-système de parties de $U^{\prime}$, l'identité (1) résulte alors du théorème de classe monotone, ref. [1] p. 41 (l'ensemble des boréliens $A \subset U^{\prime}$ tels que $y \mapsto v_{y}(A)$ est mesurable et vérifie $\Delta(K(f))(A)=\int \Delta k_{y}(A) f(y) \mathrm{d} \mu(y)$ est stable par différence propre et réunion croissante dénombrable).

\footnotetext{
1 Sans supposer $U$ borné, on a $\theta \in L^{1}\left(\mathcal{F}, \mu ; L^{2}(U)\right)$ et on aurait pu conclure plus directement avec [8] p. 198.
} 


\section{Preuve du Théorème 1}

Remarquons d'abord que les hypothèses entraînent que la mesure $\mu$ ne charge pas les parties polaires de $U$ puisque pour $A$ compact dans $U$, la mesure $1_{A} \cdot \mu$ est somme d'une mesure de la forme $\Delta v$ où $v \in H^{1}(U)$ et d'une mesure absolument continue sur $U$ (cf. la partie $\mathbf{1}$ ).

Soit $A$ une partie borélienne relativement compacte de $U$ telle que $\lambda_{d}(A)=0$. Alors si $B$ et $A^{\prime}$ sont des boréliens contenus dans $A$, on a $0=\Delta\left(K\left(1_{B}\right)\right)\left(A^{\prime}\right)-\left(1_{B} \cdot \mu\right)\left(A^{\prime}\right)$ et, par la formule du Lemme $4,0=$ $\int_{B} \Delta\left(k_{y}\right)\left(A^{\prime}\right) \mathrm{d} \mu(y)-\mu\left(B \cap A^{\prime}\right)$ où $k$ est donné par le Lemme $3-$ on a vu aussi que $\Delta k_{y}$ est une mesure pour $\mu$ presque tout $y \in U$ et que $y \mapsto \Delta k_{y}\left(A^{\prime}\right)$ est de classe $\mathcal{L}^{\infty}(\mu)-$. Ce qu'on peut écrire sous la forme :

$$
0=\int_{B}\left\{\Delta\left(k_{y}\right)\left(A^{\prime}\right)-\delta_{y}\left(A^{\prime}\right)\right\} \mathrm{d} \mu(y)
$$

où $\delta_{y}$ désigne la mesure de Dirac au point $y$. L'arbitraire sur $B$ signifie qu'en fait $\Delta k_{y}\left(A^{\prime}\right)=\delta_{y}\left(A^{\prime}\right)$ pour $\mu$ presque tout $y \in A$.

Comme $\mathcal{B}$ or $\left(\mathbb{R}^{d}\right)$ est séparable, on peut choisir une famille dénombrable $\left\{A_{j}\right\}_{j \in J}$ de parties de $A$ qui engendre la tribu des boréliens de $A$ et qui est stable par intersection finie (les $A_{j}$ forment un $\pi$-système de parties de $A$ ). L'ensemble $J$ étant dénombrable il existe un borélien $A_{0}$ de $A, \mu$ négligeable et tel que, pour tout $y \in A \backslash A_{0}$ et tout $j \in J, \Delta k_{y}$ est une mesure et $\Delta k_{y}\left(A_{j}\right)=\delta_{y}\left(A_{j}\right)$. Le théorème de classe monotone ([1] p. 41) permet alors de dire plus : si $y \in A \backslash A_{0}$, on a $\Delta k_{y}(B)=\delta_{y}(B)$ pour tout borélien $B$ contenu dans $A$.

Autrement dit, si $y \in A^{\prime}=A \backslash A_{0}$, les mesures $\Delta k_{y}$ et $\delta_{y}$ coïncident sur $A$. Or $k_{y} \in H^{1}(U)$ pour tout $y \in A^{\prime}$ et, pour ces $y,\left|\Delta k_{y}\right|$ ne peut charger les polaires ni a fortiori les points. Par conséquent, $A=A_{0}$ et $A$ est $\mu$-négligeable.

Ainsi $\mu$ ne charge pas les parties $\lambda_{d}$-négligeables de $U$ et $\mu$ est donc absolument continue par rapport à $\lambda_{d \mid U}$. Ce qui achève la démonstration.

\section{Extensions}

Il est clair que le théorème 1 s'étend à des situations bien plus générales. On énoncera ici une généralisation naturelle. Soient $E$ un espace de Banach qu'on suppose reflexif séparable ou, plus généralement, dual d'un espace de Banach séparable ${ }^{2}$. Soit aussi $L: E \rightarrow \mathcal{D}^{\prime}(U)$ une application linéaire continue (où $U$ est un ouvert de $\mathbb{R}^{d}$ ) telle que toute mesure de Radon $v$ sur $U$ appartenant à $L(E)$ soit diffuse, c'est à dire telle que $v(\{a\})=0$ pour tout $a \in U$.

Théorème 1 bis. Soient $\mu$ et $m$ deux mesures de Radon positives sur U. S'il existe un opérateur linéaire continu

$$
K: L^{1}(\mu) \rightarrow E
$$

tel que pour chaque $f \in L^{1}(\mu)$ la distribution $v_{f}=L[K(f)]$ est une mesure de Radon sur $U$ de la forme $f \cdot \mu+$ $g_{f} \cdot m, g_{f} \in L^{1}(m)$, alors $\mu$ est absolument continue par rapport à $m$.

Preuve. Puisque toute mesure appartenant à $L(E)$ est diffuse, tout point chargé par $\mu$ est chargé par $m$. Donc, quitte à remplacer $m$ et $\mu$ par leurs «parties» continues (les plus grandes mesures positives diffuses $m_{c}$ et $\mu_{c}$ majorées par $m$ et $\mu$ respectivement), on peut supposer que $m$ et $\mu$ sont diffuses. Le reste de la preuve s'obtiendra en adaptant assez directement les arguments déjà utilisés pour le Théorème 1.

\footnotetext{
2 Il suffit même pour la suite que $E$ soit le dual $F^{\prime}$-muni de la topologie faible- d'un espace localement convexe séparable $F$ à condition de supposer dans le Théorème 1 bis que $\left\{K(f) ;\|f\|_{L^{1}(\mu)} \leqslant 1\right\}$ est une partie équicontinue de $E=F^{\prime}$.
} 
Par le théorème de Dunford-Pettis, on a $K(f)=\int_{U} f(y) k_{y} \mathrm{~d} \mu(y)$ (intégrale de Bochner dans $E$ ) où $y \mapsto k_{y}$ est mesurable bornée de $U$ dans $E$. On en déduit que $\langle L(K(f)), \varphi\rangle=\int\left\langle L\left(k_{y}\right), \varphi\right\rangle f(y) \mathrm{d} \mu(y)$ pour $f \in L^{1}(\mu)$, $\varphi \in C_{0}^{\infty}(U)$. Or, comme dans le Lemme 4, pour tout ouvert relativement compact $V$ de $U$ on a $C_{V}:=$ $\sup \left\{\left|v_{f}(\varphi)\right| ;\|f\|_{L^{1}(\mu)} \leqslant 1, \varphi \in C_{0}^{\infty}(V),|\varphi| \leqslant 1\right\}<\infty$ d'après le théorème de Banach-Steinhaus. Il s'ensuit qu'il existe un borélien $\mu$-négligeable $A_{0}$ de $U$ tel que pour $y \in U \backslash A_{0}, v_{y}=L\left(k_{y}\right)$ est une mesure de Radon sur $U$ vérifiant $\left|v_{y}\right|(V) \leqslant C_{V}$. On vérifie aussi que pour chaque borélien relativement compact $A$ de $U, y \mapsto v_{y}(A)$ est mesurable (bornée) sur $U \backslash A_{0}$ et que $v_{f}(A)=\int v_{y}(A) f(y) \mathrm{d} \mu(y)$ si $f \in L^{1}(\mu)$-en considérant (par exemple) d'abord le cas de $A$ ouvert et en utilisant ensuite le théorème de classe monotone-.

Soit alors $A$ un borélien relativement compact et $m$ négligeable de $U$, disjoint de $A_{0}$. Pour $B, A^{\prime}$ boréliens de $A$, on a $0=v_{1_{B}}\left(A^{\prime}\right)-\left(1_{B} \mu\right)\left(A^{\prime}\right)$ et donc $0=\int_{B} v_{y}\left(A^{\prime}\right) \mathrm{d} \mu(y)-\mu\left(A^{\prime} \cap B\right)$ ou $0=\int_{B}\left\{v_{y}\left(A^{\prime}\right)-\delta_{y}\left(A^{\prime}\right)\right\} \mathrm{d} \mu(y)$. Faisant varier $B$, on obtient que $v_{y}\left(A^{\prime}\right)=\delta_{y}\left(A^{\prime}\right)$ pour $\mu$ presque tout $y \in A$.

Fixant un $\pi$-système $\left\{A_{j}\right\}_{j \in J}$ dénombrable et générateur de $\mathcal{B}$ or $(A)$, on obtient un borélien $\mu$-négligeable $N_{0} \subset A$ tel que $v_{y}\left(A_{j}\right)=\delta_{y}\left(A_{j}\right)$ pour $y \in A \backslash N_{0}$ et $j \in J$. Le théorème de classe monotone donne ensuite l'égalité des mesures $v_{y}$ et $\delta_{y}$ sur $A$, pour $y \in A \backslash N_{0}$. Mais $v_{y} \in L(E)$ et $\left|v_{y}\right|$ ne peut charger les points. Par conséquent, $A=N_{0}$ et $A$ est $\mu$-négligeable.

En conclusion, $\mu$ ne charge pas les parties $m$-négligeables de $U$ et $\mu$ est bien absolument continue par rapport à $m$.

Corollaire 5. Si $\mu$ est une mesure de Radon positive sur $U$ et s'il existe $K: L^{1}(\mu) \rightarrow$ E linéaire continue telle que $L(K(f))=f \mu$ pour toute $f \in L^{1}(\mu)$ alors $\mu=0$.

Remarquons enfin une amélioration «automatique » du Théorème 1 bis.

Corollaire 6. Si $\mu$ est une mesure de Radon positive et non nulle sur $U$, il n'existe pas d'application linéaire continue $K: L^{1}(\mu) \rightarrow E$ telle que pour toute $f \in L^{1}(\mu)$ la distribution $v_{f}=L(K(f))$ est une mesure de Radon sur $U$ dont la partie absolument continue relativement à $\mu$ est $f \cdot \mu$.

Preuve. Supposons $K$ comme dans l'énoncé. Comme ci-dessus, le théorème de Banach-Steinhaus montre que si on munit l'espace $\mathcal{M}(U)$ des mesures de Radon sur $U$ de la famille de semi-normes $p_{V}: \mu \mapsto|\mu|(V), V$ variant parmi les ouverts relativement compact de $U$, l'application $L \circ K: L^{1}(\mu) \rightarrow \mathcal{M}(U)$ est continue. En particulier si $\left\{f_{n}\right\}$ est une suite dense dans $L^{1}(\mu)$, si $\theta_{n}=L\left(K\left(f_{n}\right)\right)$ et si $\left\{c_{n}\right\}$ est une suite de réels $>0$ décroissant assez vite vers zéro, $\theta=\sum_{n} \geqslant 1 c_{n}\left|\theta_{n}\right|$ est une mesure de Radon positive et diffuse. De plus chaque $v_{f}, f \in L^{1}(\mu)$, est absolument continue par rapport à $\theta$.

Si maintenant $g$ désigne la plus grand élément de $L_{\text {loc }}^{1}(\mu)$ tel que $g \cdot \mu \leqslant \theta$, la mesure $m=\theta-g \cdot \mu$ est singulière par rapport à $\mu$ et telle que $v_{f}-f \mu$ est absolument continue par rapport à $m$ pour toute $f \in L^{1}(\mu)$. Le Théorème 1 bis assure alors que $\mu$ est aussi absolument continue par rapport à $m$. Et $\mu$ serait nulle contrairement à l'hypothèse.

Exemple 4.1. Prenons $E=W^{1, p}(U)$ avec $p \geqslant \frac{d}{d-1}$ et $A=\Delta$. Une mesure de Radon sur $U$ du type $v=\Delta(v)$, $v \in E$, est diffuse. Car si $B(a, r) \subset U, \varphi \in C_{0}^{\infty}(B(a, r)), 0 \leqslant \varphi \leqslant 1$ et $\varphi(a)=1$, on a

$$
v(\{a\})-v_{-}(B(a, r) \backslash\{a\}) \leqslant \int \varphi \mathrm{d} v=-\int \nabla \varphi \cdot \nabla v \mathrm{~d} \lambda_{d} .
$$

D'où, $v(\{a\})-v_{-}(B(a, r) \backslash\{a\}) \leqslant\|\nabla \varphi\|_{L^{p^{*}}}\|v\|_{W^{1, p}(B(a, r))} \leqslant C^{s t e} r^{d / p^{*}-1}\|v\|_{W^{1, p}(B(a, r))}$ pour un choix convenable de $\varphi$. Faisant tendre $r$ vers 0 , on obtient $v(\{a\}) \leqslant 0$ et donc en fait $v(\{a\})=0$. Donc le théorème 1 bis s'applique et dans le Théorème 1 , on peut remplacer $H^{1}(U)$ par $W_{\text {loc }}^{1, d /(d-1)}(U)$ et $\lambda_{d}$ par une mesure de Radon diffuse $m$ sur $U$. 
Remarque 4.2. Pour $E=W^{1, p}(U)$ avec $p<\frac{d}{d-1}$ et $L=\Delta$, la conclusion du Théorème 1 bis peut tomber en défaut. Si $\mu$ et $m$ sont deux mesures de Radon positives, à support compact dans $U$ et étrangères entre elles et si on prend $K: L^{1}(\mu) \ni f \mapsto c G(f \mu)$ où $G$ est le noyau Newtonien, $G(x, y)=|x-y|^{2-d}$, on a $\Delta K(f)=f \mu$, $\forall f \in L^{1}(\mu)$, pour $c \in \mathbb{R}$ bien choisi. (Observer que $G(v) \in E$ pour toute mesure de Radon $v$ de masse totale finie dans $U$.) Ici, la condition de « continuité » sur les $\Delta u, u \in E$, n'est pas vérifiée.

Remarque 4.3. On ne peut en général remplacer dans le Théorème 1bis l'espace $L^{1}(\mu)$ par $L^{p}(\mu)$ avec un $p>1$, même si $E=H_{0}^{1}(U)$ et $A=\Delta$. Si $K \subset U$ est un Cantor auto-similaire (du type $2^{d}$-coins par exemple) de dimension $\alpha, 0<\alpha<d$, la mesure de probabilité auto-similaire naturelle $\mu$ sur $K$ est telle que $\mu(B(x, r)) \leqslant C^{\text {ste }} r^{\alpha}$ pour $x \in \mathbb{R}^{d}, 0<r \leqslant 1$. D'après [12] p. 59, on a donc $\|\varphi\|_{L^{q}(\mu)} \leqslant C^{s t e}\|\varphi\|_{H^{1}(U)}, \forall \varphi \in C_{0}^{\infty}(U)$, si $(d-2) \frac{q}{2} \leqslant \alpha$. Mais alors $L^{q^{*}}(\mu) \subset H^{-1}(U)$ (l'injection correspondante étant continue) et l'opérateur (isomorphisme) de Green $G: H^{-1}(U) \rightarrow H_{0}^{1}(U)$ induit, pour $p>\frac{2 d}{d+2}$, une application linéaire $K: L^{p}(\mu) \rightarrow H_{0}^{1}(U)$ continue telle que $\Delta[K(f)]=f \cdot \mu$.

\section{Remerciements}

L'auteur remercie très cordialement Haïm Brezis dont les questions et l'intérêt sont à l'origine du présent travail.

\section{Références}

[1] P. Billingsley, Probability and Measure, third ed., Wiley Ser. Probab. Math. Statist., Wiley, New York, 1995.

[2] L. Boccardo, T. Gallouët, L. Orsina, Existence and uniqueness of entropy for nonlinear elliptic equations with measure data, Ann. Inst. Poincaré Anal. Non Linéaire 13 (5) (1996) 539-551.

[3] N. Bourbaki, Éléments de mathématique. XXV. Première partie. Livre VI : Intégration, Actualités Sci. Ind., vol. 1281, Hermann, Paris, 1959.

[4] J. Bourgain, H. Brezis, On the equation $\operatorname{div} Y=f$ and application to control of phases, J. Amer. Math. Soc. 16 (2) (2003) $393-426$.

[5] H. Brezis, M. Marcus, A.C. Ponce, Nonlinear elliptic equations with measures revisited, Preprint, June 2004, in press.

[6] L. Carleson, Selected Problems on Exceptional Sets, Van Nostrand Math. Stud., vol. 13, Van Nostrand, Princeton, NJ, 1967.

[7] N. Dunford, B. Pettis, Linear operations on summable functions, Trans. Amer. Math. Soc. 47 (1940) 323-392.

[8] N. Dunford, J.T. Schwartz, Linear Operators. I. General Theory, Pure Appl. Math., vol. 7, Interscience, New York, 1958.

[9] E. Gagliardo, Caratterizzazioni delle tracce sulla frontiera relative ad alcune classi di funzioni in $n$ variabili, Rend. Sem. Mat. Univ. Padova 27 (1957) 284-305.

[10] M. Grun-Rehomme, Caractérisations du sous-différentiel d'intégrandes convexes dans les espaces de Sobolev, J. Math. Pures Appl. 56 (1977) $149-156$.

[11] L.L. Helms, Introduction to Potential Theory, Pure Appl. Math., vol. XXII, Wiley, New York, 1969.

[12] V. Maz’ya, Sobolev Spaces, Springer-Verlag, Berlin, 1985.

[13] J. Peetre, A counterexample connected with Gagliardo's trace theorem, Comment. Math. 2 (1979) 277-282, special issue. 